\title{
What is the effect of dietary or supplemental micronutrient intake on sex hormones and IGF-1 in adult men and women over 45 years?
}

\author{
R. Janjuha, D. Bunn, R. Hayhoe, L. Hooper, A. Abdelhamid and A. Welch \\ Department of Population Health and Primary Care, Norwich Medical School, University of East Anglia, NR4 7TJ.
}

Age-related sarcopenia is an international problem, involving the loss of muscle mass and function. The process begins at the age of 40 years old in both males and females ${ }^{(1)}$. Currently, there are no accepted management guidelines for the condition, which has the potential to significantly adversely affect mobility, activities of daily living, and independence.

Sarcopenia is multifactorial in aetiology and one potential mechanism for its onset is the physiological age-related decline in sex hormones and insulin-like growth hormone-1 (IGF-1) ${ }^{(2)}$. Decline in these hormones occurs at a similar age to the onset of sarcopenia and micronutrients may be important in regulating their concentrations. Sex hormones and IGF-1 concentrations may be affected by oxidative stress, or by up-regulation of inflammatory cytokines, and therefore the antioxidant vitamins A, C, and E, as well as the carotenoids and zinc ( $\mathrm{Zn}$ ) may be important. Previous research has shown total antioxidant binding capacity is related to sex hormone concentration $^{(3)}$; Vitamin D increases testosterone levels in $\mathrm{men}^{(4)}$; and supplementation with some micronutrients, for example: selenium $^{(5)}(\mathrm{Se})$, or $\mathrm{Zn}^{(6)}$, increases IGF-1 levels in both men and women. However, the relationship between micronutrients and sex hormones or IGF-1 in adults over 45 years has not been extensively researched. Therefore, the aim of this systematic review (SR), is to use established Cochrane methodology, to investigate the effect of dietary or supplemental intake of specific micronutrients and associations or changes on sex hormones and IGF-1.

Only randomised controlled trials, prospective cohort studies or cross-sectional studies were eligible for inclusion in this review. Searches in MEDLINE, EMBASE and Cochrane Central Register of Controlled Trials utilised a variety of free text words, index terms and synonyms relating to: Vitamin A, C, D, E, carotenoids, minerals ( $\mathrm{Zn}$, Magnesium ( $\mathrm{Mg}$ ), Se, potassium, iron and copper), sex hormones (androgens, oestrogens, dehydroepiandrosterone (DHEAS), and sex hormone binding globulin (SHBG)), and IGF-1. A comprehensive search strategy was created after combining both micronutrient terms and terms for sex hormones. All screening, application of eligibility criteria, and data extraction will be conducted in duplicate. Studies will be considered eligible providing participants are over 45 years and without renal disease. The study protocol will be registered on PROSPERO.

A total of 5372 unique papers have been identified, and from this, 71 studies have been found to be eligible for inclusion. These studies provide data on vitamin D (44\%), multi-vitamins $(25 \%)$, Zn (9\%), vitamin E (7\%), Carotenoids (6\%), Se (4\%), Mg (3 $\%$ ) and vitamin $\mathrm{C}(1 \%)$. Data extraction has yet to commence, however, the analysis will include a narrative synthesis and meta-analyses if this is viable with the final data. The majority of studies on micronutrients are cross-sectional in design (55\%), with remaining as randomised (37\%) or cohort studies $(8 \%)$. The studies report a range of outcomes, the majority including IGF-1.

The findings of this SR will further our understanding of the influence of oral micronutrient intake on sex hormone and IGF-1 concentrations. This is expected to be important in informing the development of management strategies for sarcopenia.

1. Marzetti E, Calvani R, Tosato M, et al. (2017) Aging Clin Exp Res. 29(1), 11-7.

2. Maggio M, Lauretani F \& Ceda GP. (2013) Curr Opin Clin Nutr Metab Care. 16(1):3-13.

3. Demirbag R, Yilmaz R \& Erel O. (2005) Scand Cardiovasc J. 39(3):172-6.

4. Pilz S, Frisch S, Koertke $\mathrm{H}$ et al. (2011) Hormones et metabolisme. 43(3), 223-5.

5. Maggio M, Ceda GP, Lauretani F et al. (2010) Clin Nutr. 29(5), 674-7.

6. Rodondi A, Ammann P, Ghilardi-Beuret S \& Rizzoli R. (2009) The journal of nutrition, health \& ageing 13(6), 491-7. 